\title{
INCREMENTAL SEMI-SUPERVISED CLUSTERING Method Using Neighbourhood AssignMENT
}

\author{
P. Ganesh Kumar ${ }^{1}$ and A.P.Siva Kumar ${ }^{2}$ \\ ${ }^{1}$ Department of Computer Science and Engineering, JNTUA University, Anantapur, India \\ ${ }^{2}$ Assistant Professor, JNTUA, Anantapur, India
}

\begin{abstract}
Semi-supervised considering so as to cluster expects to enhance clustering execution client supervision as pair wise imperatives. In this paper, we contemplate the dynamic learning issue of selecting pair wise must-connect and can't interface imperatives for semi supervised clustering. We consider dynamic learning in an iterative way where in every emphasis questions are chosen in light of the current clustering arrangement and the current requirement set. We apply a general system that expands on the idea of Neighbourhood, where Neighbourhoods contain "named samples" of distinctive bunches as indicated by the pair wise imperatives. Our dynamic learning strategy extends the areas by selecting educational focuses and questioning their association with the areas. Under this system, we expand on the fantastic vulnerability based rule and present a novel methodology for figuring the instability related with every information point. We further present a determination foundation that exchanges off the measure of vulnerability of every information point with the expected number of inquiries (the expense) needed to determine this instability. This permits us to choose questions that have the most astounding data rate. We assess the proposed strategy on the benchmark information sets and the outcomes show predictable and significant upgrades over the current cutting edge.
\end{abstract}

\section{KEYWORDS}

Active learning, clustering, semi-supervised learning

\section{INTRODUCTION}

SEMI-SUPERVISED clustering intends to enhance clustering execution with the assistance of client gave side data. A standout amongst the most concentrated on sorts of side data is pair wise limitations, which incorporate must link what's more, can't connection requirements indicating that two focuses must or must not have a place with the same group. Various past studies have exhibited that, by and large, such imperatives can prompt enhanced clustering execution . On the other hand, if the imperatives are chosen shamefully, they might likewise corrupt the clustering execution. Besides, acquiring pair wise imperatives regularly obliges a client to physically review the information focuses being referred to, which can be tedious and excessive. For instance, for report clustering, acquiring an absolute necessity join then again can't connect limitation obliges a client to conceivably examine through the reports being referred to and focus their relationship, which is achievable yet unreasonable in time. For those reasons, we might want to upgrade the choice of the imperatives for semi-supervised clustering, which is the theme of dynamic learning. While dynamic learning has been widely concentrated on in supervised learning [6], [7], [8], [9], [10], [11], the examination on dynamic learning of requirements for semi-supervised clustering is

DOI : 10.5121/ijcsea.2016.6202 
generally constrained [1], [5], [12], [13], [14]. A large portion of the existing chip away at this theme has concentrated on selecting a beginning set of requirements preceding performing semisupervised clustering [1], [5], [13], [14]. This is not suitable in the event that we wish to iteratively enhance the clustering model by effectively questioning the client. In this paper, we consider dynamic learning of requirements in an iterative structure. In particular, in every cycle we figure out what is the most critical data toward enhancing the present clustering model and structure inquiries likewise. The reactions to the questions (i.e., limitations) are then used to redesign (and enhance) the clustering. This procedure rehashes until we achieve an acceptable arrangement or we achieve the greatest number of inquiries permitted. Such an iterative system is broadly utilized as a part of dynamic learning for supervised characterization [7], [8], [9], [10], and has been by and large saw to beat noniterative strategies, where the entire arrangement of inquiries is chosen in a solitary bunch. We concentrate on a general methodology in view of the idea of neighbourhoods, which has been effectively utilized as a part of a number of past studies on dynamic obtaining of limitations [1], [12], [13]. An area contains an arrangement of information directs that are known toward fit in with the same group as per the requirements and distinctive neighbourhoods are known not to distinctive groups. Basically, Neighbourhoods can be seen as containing the "named illustrations" of distinctive groups. Very much shaped Neighbourhoods can give important data with respect to what the hidden bunches resemble. Comparable to supervised dynamic learning, a dynamic learner of imperatives will then try to choose the most enlightening information point to incorporate in the areas. When a point is chosen, we question the chosen point against the current Neighbourhoods to focus to which Neighbourhood it has a place. In particular, our methodology expands on the exemplary vulnerability based rule. Here, we characterize the vulnerability in terms of the likelihood of the point having a place with diverse known Neighbourhoods and propose a novel nonparametric methodology utilizing irregular woods [15] for assessing the probabilities. Unique in relation to supervised learning where every point just obliges one question to get its mark, in IEEE TRANSACTIONS ON KNOWLEDGE AND DATA ENGINEERING, VOL. 26, NO. 1, JANUARY 201443 1041-4347/14/\$31.00 2014 IEEE Published by the IEEE Computer Society semi-supervised clustering, we can just posture pair wise questions and it regularly takes various inquiries to focus the area of a chose point. By and large, focuses with higher vulnerability will oblige bigger number of questions. This proposes that there is tradeoffs between the measure of data we gain by questioning around a point, and the expected number of inquiries (expense) for procuring this data. We propose to adjust this tradeoffs by normalizing the measure of instability of every information point by the normal number of inquiries needed to determine this instability, and as being what is indicated, select inquiries that have the most elevated rate of data.

Note that an undeniable option methodology would be to assess every potential match and select the particular case that has the most astounding vulnerability in regards to whether they are mustconnected on the other hand can't connected. This thought has beforehand been investigated by Huang and Lam [12] in the connection of archive clustering.

In this paper, we take note of a discriminating issue with this approach that it just considers the pair wise instability of the first question what's more, neglects to quantify the advantage of the resulting questions that are obliged to focus the area for a point. Our system, rather, concentrates on the point-based vulnerability, permitting us to choose the inquiries as indicated by the aggregate measure of data picked up by the full grouping of inquiries all in all. We exactly assess the proposed strategy on eight information sets of distinctive unpredictability. The assessment results show that our strategy accomplishes steady and significant enhancements more than three contending routines. 


\section{RELATED WORK}

Dynamic learning has been contemplated widely for supervised arrangement issues [6], [7], [8], [9], [10], [11]. As said beforehand, most of the current examination concentrated on the determination of an arrangement of initial constraints prior $\mathrm{t}$ o performing semi-supervised clustering. In particular, the first study on this subject was led by Basu et al. [1]. They proposed a two-stage approach, which we allude to as the Explore and Consolidate (E \& C) approach. The main stage (Explore) incrementally chooses focuses utilizing the most remote first traversal plan and questions their relationship to distinguish c disjoint Neighbourhoods, where $\mathrm{c}$ is the aggregate number of bunches. The second stage (merge) iteratively grows the areas, where in every cycle it chooses an irregular point outside any area and questions it against the current Neighbourhoods until an unquestionable requirement connection is found. All the more as of late, Mallapragada et al. [13] proposed a change to Investigate and Consolidate named Min-Max, which changes the merge stage by picking the most dubious point to question (instead of arbitrarily).

$\mathrm{Xu}$ et al. [14] proposed to choose imperatives by inspecting the ghostly eigenvectors of the closeness network, which is lamentably constrained to two-group issues. In [5], [16], imperatives are chosen by examining the co-affiliation lattice (acquired by applying group outfits to the information). A key refinement of our technique from the aforementioned work is that we iteratively select the following arrangement of questions taking into account the present clustering task to enhance the arrangement. This is closely resembling supervised dynamic learning where information focuses are chosen iteratively taking into account the current characterization model such that the model can be enhanced most effectively [7], [8], [9], [10]. More applicable to our work is a dynamic learning structure exhibited by Huang and Lam [12] for the errand of record clustering. In particular, this structure takes an iterative approach that is like our own. In each emphasis, their system performs semi-supervised clustering with the present arrangement of limitations to deliver a probabilistic clustering task. It then processes, for every pair of archives, the likelihood of them having a place to the same bunch and measures the related instability. To make a determination, it concentrates on all unconstrained sets that has precisely one archive officially "allocated to" one of the current Neighbourhoods by the present limitation set, and among them recognizes the most unverifiable pair to inquiry. On the off chance that an "absolute necessity connection" answer is returned, it stops and moves onto the following emphasis. Else, it will inquiry the unassigned point against the current Neighbourhoods until an "absolute necessity connection" is returned. While Huang's technique is created particularly for report clustering, one could possibly apply the hidden dynamic learning way to deal with handle different sorts of information by expecting proper probabilistic models. We might want to highlight a key refinement between Huang's technique and our work, that is Huang's strategy makes the determination decision in light of pairwise instability, while we concentrate on the vulnerability of a point regarding which Neighbourhood it has a place with. This distinction is unobtrusive, yet imperative. Pairwise instability catches just the relationship between the two focuses in the pair. Contingent upon the result of the question, we may need to experience a arrangement of extra questions. Huang's technique just considers the pairwise vulnerability of the first question, and neglects to quantify the advantage of the resulting questions. This is why our system rather concentrates on point-based vulnerability, which measures the aggregate sum of data picked up by the full succession of inquiries in general. Besides, our strategy likewise considers the anticipated that number of questions would resolve the instability of a point, which has not been considered beforehand. At long last, we need to say a different profession that utilizes dynamic learning to encourage clustering [17], [18], where the objective is to group a situated of articles by effectively questioning the separations between one or more combines of focuses. This is not 
the same as the centre of this paper, where we just demand pair wise must-connect and cannot link imperatives, and don't require the client to give particular separation values.

\section{METHODOLOGY}

The issue tended to in this paper is the means by which to viably pick pairwise inquiries to deliver an exact clustering task. Through dynamic learning, we plan to accomplish inquiry effectiveness, i.e., we might want to diminish the quantity of inquiries/inquiries requested that accomplish a decent clustering execution. We see this as an iterative process such that the choice for selecting questions ought to rely on upon what has been gained from the all the detailed inquiries. In this segment, we will present our proposed strategy. Underneath, we will start by giving an exact plan of our dynamic learning issue.

\subsection{Problem Formulation}

Formally, we characterize the issue as takes after: given an arrangement of information occasions D $1 / 4 \mathrm{f} \mathrm{x} 1 ; \ldots$; xng, we expect that there exists a basic class structure that relegates every information example to one of the c classes. We signify the obscure marks by y $1 / 4$ fy $1 ; \ldots$; yng, every mark yi 2 Y $1 / 44 \mathrm{f} 1 ; \ldots ; c g, 8 \mathrm{i} 2 \mathrm{f} 1 ; \ldots$; ng. In this setting, we can't (straightforwardly) watch these marks. Rather, data can be acquired through question of the structure: Do cases xi and $x j$ have a place with the same class? We signify a question by a couple of occasions $\partial x i$; xjp, and the response to the question by lij 2 A $1 / 44 \mathrm{fML}$; CLg. Specifically, the name "ML" ("CL") is returned if yi $1 / 4$ yj (yi $61 / 4 \mathrm{yj}$ ). In every cycle, we have to choose one or more questions in view of $\mathrm{D}$ and the present arrangement of imperatives C. Note that must-interface and can't connect requirements fulfill the accompanying properties:

Taking into account these properties, we present the idea of Neighbourhood, which is instrumental in the outline of numerous existing routines for dynamic learning of pair wise limitation.

\subsection{Neighbourhood-Based Framework}

Definition 1. An area contains an arrangement of information occasions that are known not to the same class (i.e., associated by must-connect limitations). Moreover, distinctive Neighbourhoods are associated by can't connect limitations and, accordingly, are known to have a place with diverse classes.

Given an arrangement of limitations meant by $\mathrm{C}$, we can distinguish a set of 1 Neighbourhoods $\mathrm{N}$ $1 / 4 \mathrm{fN} 1 ; \ldots ; \mathrm{Nlg}$, such that $1 \mathrm{c}$ and $\mathrm{c}$ is the aggregate number of classes. Consider a diagram representation of the information where vertices speak to information cases, and edges speak to must-interface imperatives. The Neighbourhoods, which are meant by Ni D; i 2 f1; . .; lg, are just the associated segments of the chart that have can't interface limitations between each other. Note that on the off chance that there exists no can't connect imperatives, we can just distinguish a solitary known Neighbourhood despite the fact that we might have different joined parts in light of the fact that some associated segments may fit in with the same class. In such cases, we will regard the biggest joined segment as the known Neighbourhood. 


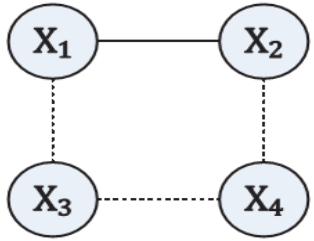

(a)

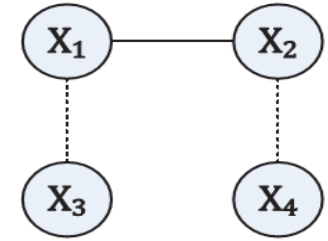

(b)

Figure 1 represents two samples that clarify how we can

structure the areas from an arrangement of pairwise imperatives. The hubs mean information examples, and the strong lines indicate must-connect limitations while the dashed lines signify cannotlink imperatives. Note that in our definition, every area is obliged to have a can't interface imperative with all different Neighbourhoods. Consequently, Fig. 1a contains three Neighbourhoods: fx1; $\mathrm{x} 2 \mathrm{~g}$; fx3g, and $\mathrm{fx} 4 \mathrm{~g}$, while Fig. $1 \mathrm{~b}$ contains just two known Neighbourhoods, which can be either $\mathrm{fx} 1 ; \mathrm{x} 2 \mathrm{~g}$; fx3g or fx1; $2 \mathrm{~g}$; f $\mathrm{f} 4 \mathrm{~g}$. One approach to translate the areas is to view them as the "marked cases" of the hidden classes on the grounds that occasions having a place with distinctive Neighbourhoods are ensured to have diverse class marks, and occurrences of the same Neighbourhood must fit in with the same class. A key point of preference of utilizing the area ideas is that by utilizing the information of the areas, we can gain a substantial number of requirements by means of a little number of inquiries. Specifically, in the event that we can distinguish the area of an occasion $\mathrm{x}$, we can promptly construe its pairwise association with every single other point that are at present affirmed to have a place with any of the current Neighbourhoods. This actually persuades us to consider a dynamic learning system that incrementally extends the areas by selecting the most enlightening information point and questioning it against the known Neighbourhoods. We compress this system in Algorithm 1.

Calculation 1. The Neighbourhood-based Framework

Info: An arrangement of information focuses D; the aggregate number of classes c; the greatest number of pairwise inquiries $T$.

Yield: a clustering of D into c bunches.

1: Initializations: $\mathrm{C} 1 / 4 ; ; \mathrm{N} 1 \frac{1 / 4}{\mathrm{fxg}}$, where $\mathrm{x}$ is an irregular point in $\mathrm{D} ; \mathrm{N} 1 \frac{1}{4} \mathrm{~N} 1 ; 1 \frac{1}{4} 1 ; \mathrm{t}^{1 / 4} 0$;

2: rehash

3: 1/4 Semi-supervised-Clustering $(\mathrm{D}, \mathrm{C})$;

4: $\mathrm{x}$

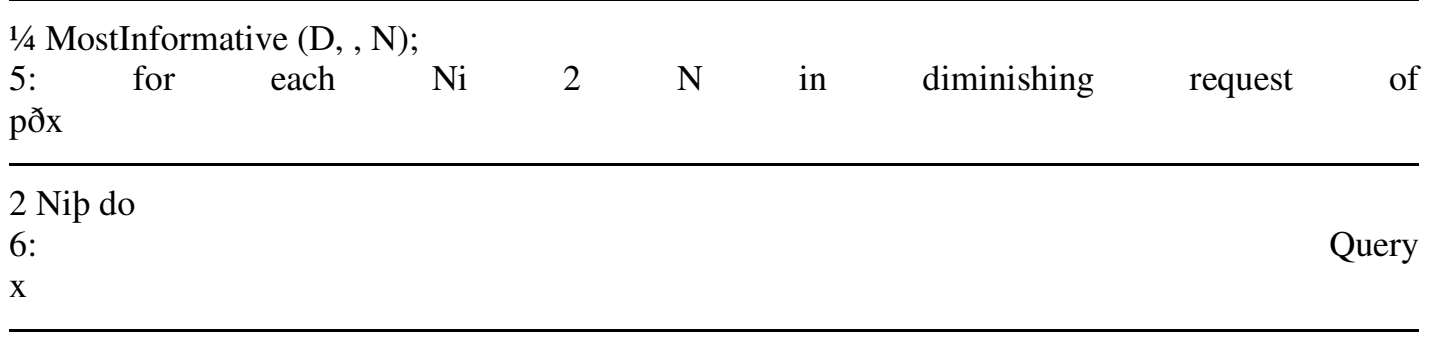

against any information point xi $2 \mathrm{Ni}$;

7: t p;

8: Update $\mathrm{C}$ in view of returned answer; 
ðx

\begin{tabular}{llllllll}
\hline$;$ & xi; & MLP & then & Ni & $1 / 4$ & Ni & [ \\
\hline
\end{tabular}

$\mathrm{g}$; break;

10: end for

11: if no must-connection is accomplished

12.

fx

g; $\mathrm{N}^{1} / 4 \mathrm{~N} \mathrm{~S} \mathrm{Nl}$

13: until $\mathrm{t}>\mathrm{T}$

14: arrival Semi-supervised-clustering(D, C)

Quickly, the calculations start by selecting so as to instat the areas an arbitrary point to be the beginning Neighbourhood (line 1). In every emphasis, given the current set of requirements $\mathrm{C}$, it performs semi-supervised clustering on D to create a clustering arrangement (line 3). A choice measure is then connected to choose the "most instructive" information point $\mathrm{x}$

in light of the present arrangement of Neighbourhoods and the clustering arrangement (line 4). The chose point $\mathrm{X}$

is at that point questioned against every current Neighbourhood $\mathrm{Ni}$ to recognize where $\mathrm{x}$

has a place, amid which the limitation set $\mathrm{C}$ Fig. 1. Two cases to demonstrate to distinguish Neighbourhoods from a set of pairwise requirements. is redesigned (lines 5-12). In line 5, we experience the Neighbourhoods in diminishing request in view of $p d x$

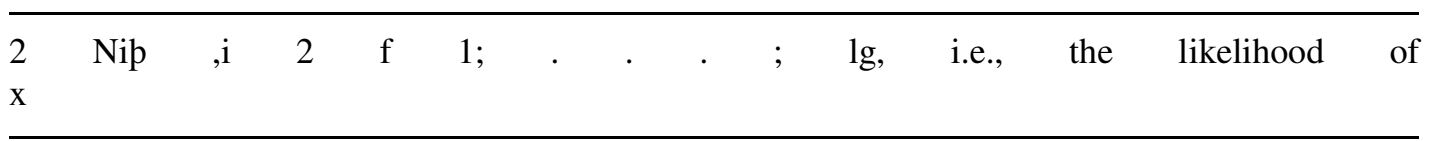

having a place with each Neighbourhood, which is thought to be known. This inquiry request will permit us to focus the area of $\mathrm{x}$

with the littlest number of inquiries. This procedure is rehashed until we achieve the most extreme number of inquiries permitted (line 13).

In this work, we consider the semi-supervised clustering calculation as a black box and any current calculation can be utilized here. The key inquiry we expect to answer is the way to select the "most educational" example to inquiry against, i.e., the outline of the capacity MostInformative in line 4. In the remaining piece of this area, we will concentrate on this inquiry what's more, portray our program 


\subsection{Normalizing Uncertainty with Expected Cost}

Note that we inquiry a chose occurrence against the current Neighbourhoods to focus to which Neighbourhood it has a place. Given a chose information occurrence, it may take various pairwise inquiries to choose its Neighbourhood. In our choice measure, we ought to think seriously about this. Specifically, we can consider the quantity of inquiries needed to achieve an absolute necessity join as the expense connected with every information occasion. To characterize and measure this cost more unequivocally, give us a chance to investigate the questioning procedure. Given a chose occasion $\mathrm{x}$, and the probabilities of it fitting in with diverse Neighbourhoods, which Neighbourhood should we inquiry against first? Expect the evaluated probabilities pð x 2 $\mathrm{Nip}$ are precise for all $\mathrm{x} 2 \mathrm{D}$ and $\mathrm{Ni} 2 \mathrm{~N}$, we ought to dependably begin by questioning $\mathrm{x}$ against the Neighbourhood that has the most elevated likelihood of containing $\mathrm{x}$ to minimize the aggregate number of obliged inquiries. In the event that a must-connection is returned, we can stop with stand out inquiry. Something else, one ought to ask the following inquiry against the Neighbourhood that has the following most elevated likelihood of containing $\mathrm{x}$. This technique is rehashed until an absolute necessity join requirement is returned or we have a can't connect imperative against all areas, and soon thereafter another Neighbourhood will be made utilizing $\mathrm{x}$. Let qð xp signify the irregular variable of the aggregate number of questions that we have to focus the area participation of $\mathrm{x}$. Expecting that the areas are positioned in view of their likelihood of containing $\mathrm{x}$ in plunging request, i.e., pð x $2 \mathrm{~N} 1 \mathrm{p}$ pð x $2 \mathrm{~N} 2 \mathrm{p}$ pð x $2 \mathrm{Nlp}$, where 1 is the aggregate number of existing Neighbourhoods, it is clear to demonstrate that pðqð xp 1/4 ip 1/4 pð x 2 Nip. The desire IE $1 / 2 q$ x xpis, therefore, processed by the taking after mathematical $\begin{array}{llllllll}\text { statement: } & I^{1} / 2 & 1 / 4 \mathrm{q} \gamma & \mathrm{xp} & \mathrm{X} & 1 & \mathrm{i}^{1} / 41 & \mathrm{i}\end{array}$

pð x $2 \mathrm{Nip}$; ð3p where 1 is the aggregate number of existing Neighbourhoods. On the off chance that we consider HðN j xp, the entropy of the area participation of $\mathrm{x}$ (characterized by (2)), as the measure of data we pick up by questioning about information example $x, I^{1} / 2 q$ ð xpis just the expense for acquiring this data as measured by the quantity of questions expended. In a perfect world, we might want to augment the increase of data, i.e., HðN j xp, and in the meantime minimize the expense, i.e., IE $1 / 2 q \partial \mathrm{xp}$. On the other hand, these two targets are inconsistent and we exchange off them by selecting the occurrence that amplifies the proportion between them, $\mathrm{x}$

$1 / 4 \operatorname{argmax} x 2 \mathrm{U}$ HðN j xp IE $1 / 2$ q $\mathrm{xp}$; ð4p where $\mathrm{U}$ indicates the arrangement of unconstrained cases (i.e., the set of focuses that don't fit in with any area). This basis can be translated as selecting the example that has the most elevated rate of data per question. In this way, we have depicted our proposed technique for selecting the most useful case to question. We outline this determination calculation in Algorithm 2. This finishes the portrayal of our general calculation which is outlined in Algorithm 1.

Calculation 2. MostInformative(D, N).

Information: An arrangement of information cases D; the bunch assignments ;

An arrangement of Neighbourhoods N 1/4 Sli1/41 Ni;

Yield: The most useful information point

$\mathrm{X}$ 
1: Learn an arbitrary woods classifier on D0 $1 / 4 \mathrm{f} \mathrm{xi}$; $ð$ xipgni $1 / 41$, also, register the likeness framework M;

2: for every $\mathrm{x} 2 \mathrm{D}$, and $62 \mathrm{Sli} 1 / 41 \mathrm{Ni}$ do

3: for $\mathrm{i} 1 / 4$ to 1 do

4: Compute pð x 2 Nip utilizing (1);

5: end for

6: Compute HðN j xp utilizing (2);

7: Compute IE $1 / 2 q$ ð xp utilizing (3);

8: end for

9:Return

$\mathrm{x}$

1/4 arg maxx2U HIEðN j1²qðxxPP where U 1/4 D n Sli1/41 Ni

3.4 Runt ime Analysis

In this area, we break down the runtime of our proposed calculation. Specifically, we will concentrate on Algorithm 2 since it is the center piece of our dynamic learning calculation. In line 1 , we construct an irregular woodland classifier, whose running time is $\mathrm{O}$ ðNTn log np ,3 where NT is the quantity of choice trees in RF and $n$ is the quantity of occurrences in the information [19]. Once the RF classifier is manufactured, developing a full comparability lattice will take Oðn 2 p. In any case, we needn't bother with to evaluate the full similitude framework, rather we just need to gauge a subset of the grid of size $m n$, where $m$ is the aggregate number of focuses in the areas. As a result, the aggregate runtime of line 1 is OðNTn $\log n \mathrm{p} n m p$. The for-circle in line 2 is executed at most Oðnp times, and the runtime of every execution is Oðm p cp, where $m$ is the aggregate number of "named" examples, i.e., the occurrences that are doled out to a known Neighbourhood. We can for the most part bound both $\mathrm{m}$ and $\mathrm{c}$ by $\mathrm{T}$, the aggregate number of inquiries permitted to ask, on the grounds that it takes no less than one inquiry to appoint an occurrence to an area and $\mathrm{T}$ is for the most part more noteworthy than c. In this manner, we can bound the aggregate runtime between line $2-8$ by OðnTP. Assembling it, the aggregate runtime of Algorithm 2 is OðNTn $\log n$ p nTP. Exactly, with a nonoptimized Matlab execution on an Intel 8Core i7-2600 CPU at $3.40 \mathrm{GHz}$, the normal time to choose an occurrence to inquiry for the biggest information set we tried different things with (i.e., Digits- 389 with 3,165 occurrences) is give or take 0.02 second (utilizing irregular woods of 50 choice trees). For altogether bigger information sets with a large number of occurrences and a great many highlights, extra systems could be connected to scale up our system. Case in point, the irregular timberland learning step can be effectively parallelized to expand the proficiency. Another probability would be to create and apply an incrementally when new constraints are incorporated

\section{EXPERIMENTAL SETUP}

\subsection{Data Sets}

In our trials, we utilize eight benchmark UCI information sets [21] that have been utilized as a part of past studies on constraintbased clustering [1], [4]. Out information sets incorporate bosom [22], pen-based acknowledgment of written by hand digits $(3,8,9)$, ecoli, glass distinguishing proof, statlog-heart, parkinsons [23], statlogimage division, and wine. For the ecoli information set, we uprooted the littlest three classes, which just contain 2, 2, what's more, 5 occurrences, separately. The qualities of the eight information sets are indicated in Table 1. 


\subsubsection{Experimental Setting}

Our dynamic learning structure accept the accessibility of a limitation based clustering calculation. For this reason, we utilize the surely understood MPCKMeans [3] calculation, as actualized in the WekaUT bundle [24]. We set the most extreme number of cycles of MPCKmeans to 200, also, utilized default values for different parameters. Note that the decision of this calculation is not discriminating and our system can be utilized with any requirement based clustering calculation.

At the point when assessing the execution of a specific strategy on a given information set $\mathrm{D}$, we apply it to choose up to 150 pairwise inquiries, beginning from no limitation by any stretch of the imagination. The inquiries are addressed in view of the ground-truth class name for the information set. MPCKmeans is then connected to the information with the coming about limitations (and their transitive terminations). To represent the irregularity in both dynamic learning and MPCKmeans, we rehash this procedure for 50 free runs and report the normal execution utilizing assessment criteria depicted underneath.

\subsubsection{Evaluate particle Criteria}

Two assessment criteria are utilized as a part of our analyses. To begin with, we utilize standardized common data (NMI) to assess the clustering assignments against the ground-truth class marks [25]. NMI considers both the class name and clustering task as irregular variables, and measures the common data between the two arbitrary variables, what's more, standardizes it to a zero-to-one territory. All in all, leave $\mathrm{C}$ alone the irregular variable speaking to the bunch assignments of examples, and $\mathrm{K}$ be the irregular variable speaking to the class marks of the examples, the NMI is figured by the taking after mathematical statement: NMI 1/4 2IðC; Kp НðCP p НðKР ;

where IðX; $\mathrm{Yp} 1 / 4 \mathrm{H \partial XP} \mathrm{H \partial X} \mathrm{j} \mathrm{Yp} \mathrm{is} \mathrm{the} \mathrm{common} \mathrm{data} \mathrm{between} \mathrm{irregular} \mathrm{variables} \mathrm{X}$ and $\mathrm{Y}$. HðXР is the entropy of $\mathrm{X}$, and $\mathrm{HðX} \mathrm{j} \mathrm{Yp}$ is the restrictive entropy $\mathrm{X}$ given $\mathrm{Y}$. Second, we consider F-measure as another paradigm to assess how well we can foresee the pairwise relationship between every pair of examples in examination to the relationship characterized by the ground-truth class marks [1]. F-measure is characterized as the symphonies mean of exactness and review, which are computed by the following equations

$$
\begin{aligned}
\text { Precision } & =\frac{\# \text { PairsCorrectlyPredictedInSameCluster }}{\# \text { TotalPairsPredictedInSameCluster }}, \\
\text { Recall } & =\frac{\# \text { PairsCorrectlyPredictedInSameCluster }}{\# \text { TotalPairsActuallyInSameCluster }}, \\
\text { F-measure } & =\frac{2 \times \text { Precision } \times \text { Recall }}{\text { Precision }+ \text { Recall }} .
\end{aligned}
$$

\subsection{Experimental Results}

This area exhibits the examination results, which contrast our proposed system with the gauge strategies. In the remaining discourse, we will allude to our strategy as the standardized pointbased vulnerability (NPU) strategy. 


\subsubsection{Evaluate particle Based on Clustering Performance}

The NMI estimations of NPU and the pattern strategies are demonstrated in Fig. 2. The x-pivot demonstrates the aggregate number of pairwise questions and the y-hub demonstrates the subsequent clustering execution (as measured by NMI) by running MPCKmeans with the imperatives came back from the inquiries (and their transitive terminations). As specified already, every bend demonstrates the normal execution of a technique over 50 autonomous arbitrary runs. The blunder bar on every information point shows the certainty interim (t-test at 95 percent importance level). Note that we utilize around 150 questions for all be that as it may, two information sets, in particular bosom and wine. For these two information sets, NPU meets before spending 150 questions, accordingly we demonstrate the outcomes up to 100 inquiries. From Fig. 2 , we can see that the requirements chose by NPU for the most part prompts clustering results that are more steady with the hidden class marks, as can be seen by the ruling bend of NPU contrasted with other benchmark bends. It is fascinating to note that irregular really debases the execution in some information sets as we incorporate more limitations, specifically the bosom, heart, and wine information sets. Past studies on semi-supervised clustering [4], [5], [26] have reported comparable results, where haphazardly chose requirements really debases the clustering execution for some information sets. This further shows the significance of selecting the right arrangement of requirements. In correlation, Min-Max and Huang's strategies are for the most part ready to enhance the execution reliably as we expand the quantity of inquiries, yet their execution are overwhelmed by NPU much of the time.

We additionally take note of that in the early stages, the execution of the three nonrandom systems are genuinely close. As we build the quantity of questions, the execution advantage of our technique turns out to be more declared. This is expected on the grounds that our system make more unequivocal utilization of the present clustering arrangement when selecting the inquiries. As we expand the quantity of questions, the clustering arrangement will turn out to be better and better, prompting more purported execution point of interest of our system. 4.2.2 Evaluat particle Based on Pairwi se Relation ship F-measure concentrates on how precisely we can anticipate the pairwise relationship between any pair of occurrences. In Table 2, we demonstrate the F-measure qualities accomplished by diverse strategies with inquiry sizes of 20, $40,60,80$, and 100 . For every question size, we analyze distinctive strategies against one another utilizing matched t-test at 95 percent centrality level and the best performing method(s) are at that point highlighted in boldface. At long last, Table 3 gives a rundown of the win/tie/misfortune numbers of the proposed technique versus alternate strategies. This arrangement of results are fundamentally the same to what we watch at the point when assessing utilizing NMI. At the point when utilizing just 20 questions, the execution of the nonrandom techniques frequently don't exhibit measurably noteworthy contrast. On the other hand, as we expand the quantity of questions, our strategy starts to overwhelm every single other strategy.

\subsubsection{Further Analysis of Results}

Beneath we give some more inside and out examination of the execution to comprehend what are the key elements adding to the execution favorable position of our system. With or without investigate. In the Min-Max technique, the first stage is Explore, which utilizes uttermost first traversal to discover no less than one illustration from every area to acquire a great "skeleton" of the bunches. Basu et al. [1] demonstrated that given an arrangement of c disjoint balls (bunches) of uneven sizes, Investigate is ensured to get no less than one illustration from each bunch with a little number of questions. Our system does not utilize a different Explore stage to intentionally assemble c Neighbourhoods. Does this help or hurt our execution?To answer this inquiry, we 
International Journal of Computer Science, Engineering and Applications (IJCSEA) Vol.6, No.2, April 2016

consider a two-stage variation of NPU, which performs Explore first (as utilized by Min-Max), trailed by the NPU choice foundation.
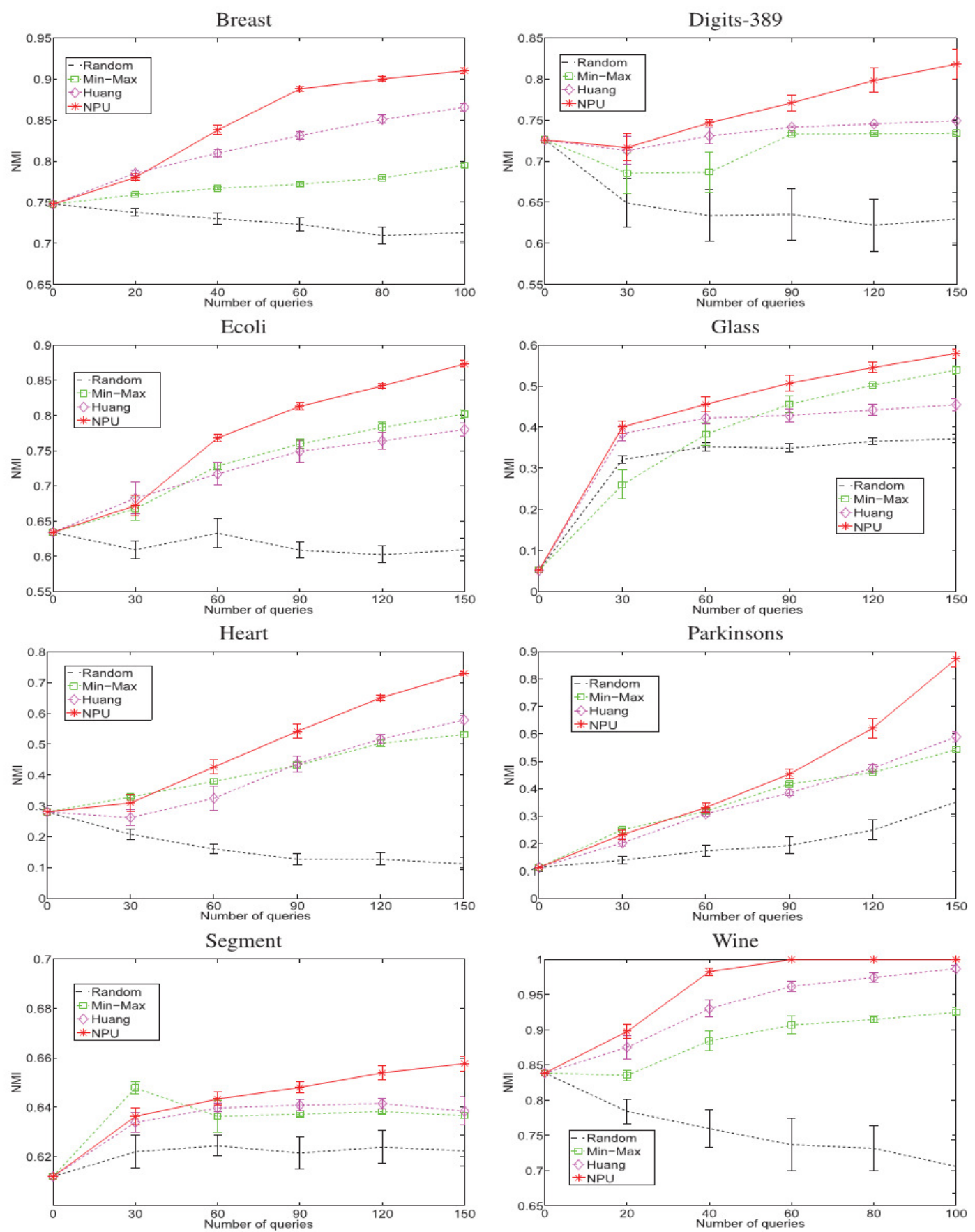

Figure 2. The NMI values of different methods on eight data sets as a function of the number of pair wise queries 


\section{CONCLUSIONS AND FUTURE WORK}

In this paper, we mull over an iterative dynamic learning structure to choose pair wise requirements for semi-supervised clustering and propose a novel system for selecting the most enlightening questions. Our system takes an area based methodology, and incrementally grows the areas by posturing pairwise inquiries. We devise a case based choice rule that distinguishes in every cycle the best occasion to include into the existing Neighbourhoods. The selection paradigm exchanges off two variables, the data substance of the example, which is measured by the instability about which Neighbourhood the example fits in with; and the expense of air conditioning qui ring this inform at I o n, which is measured by the expected number of questions needed to focus its Neighbourhood.

We observationally assess the proposed system on the eight benchmark information sets against various contending techniques. The assessment results show that our strategy accomplishes reliable and significant upgrades over its contenders. There are various intriguing bearings to expand reclustering of the information with an incrementally developing requirement set. This can be computationally requesting for huge information sets. To address this issue, it would be interesting to consider an incremental semi-supervised clustering met hodthatupdtes the ex is tin $\mathrm{g}$ clustering arrangement in light of the area task for the new point. An option approach to bring down the computational expense is to diminish the quantity of emphases by applying a clump approach that chooses an arrangement of focuses to inquiry in each emphasis. A guileless bunch dynamic learning methodology would be to choose the top $\mathrm{k}$ focuses that have the most astounding standardized instability to inquiry their Neighbourhoods. Then again, such a technique will commonly choose very repetitive focuses. Planning a fruitful bunch system requires deliberately exchanging off the quality (standardized instability) of the chosen focuses and the assorted qualities among them—a bearing that we plan to seek after for future work.

\section{REFERENCES}

[1] S. Basu, A. Banerjee, and R. Mooney, "Active Semi-Supervision for Pairwise Constrained Clustering,” Proc. SIAM Int'l Conf. Data Mining, pp. 333-344, 2004.

[2] S. Basu, I. Davidson, and K. Wagstaff, Constrained Clustering:Advances in Algorithms, Theory, and Applications. Chapman \& Hall,2008.

[3] M. Bilenko, S. Basu, and R. Mooney, "Integrating Constraints and Metric Learning in SemiSupervised Clustering," Proc. Int'l Conf. Machine Learning, pp. 11-18, 2004.

[4] I. Davidson, K. Wagstaff, and S. Basu, "Measuring Constraint-Set Utility for Partitional Clustering Algorithms," Proc. 10th European Conf. Principle and Practice of Knowledge Discovery in Databases,pp. 115-126, 2006.

[5] D. Greene and P. Cunningham, "Constraint Selection by Committee: An Ensemble Approach to Identifying Informative Constraints for Semi-Supervised Clustering," Proc. 18th European Conf. Machine Learning, pp. 140-151, 2007.

[6] D. Cohn, Z. Ghahramani, and M. Jordan, “Active Learning with Statistical Models,” J. Artificial Intelligence Research, vol. 4, pp. 129-145, 1996.

[7] Y. Guo and D. Schuurmans, "Discriminative Batch Mode Active Learning," Proc. Advances in Neural Information Processing Systems, pp. 593-600, 2008.

[8] S. Hoi, R. Jin, J. Zhu, and M. Lyu, "Batch Mode Active Learning and Its Application to Medical Image Classification,” Proc. 23rd Int'l Conf. Machine learning, pp. 417-424, 2006.

[9] S. Hoi, R. Jin, J. Zhu, and M. Lyu, "Semi-Supervised SVM Batch Mode Active Learning for Image Retrieval," Proc. IEEE Conf. Computer Vision and Pattern Recognition, pp. 1-7, 2008.

[10] S. Huang, R. Jin, and Z. Zhou, "Active Learning by Querying Informative and Representative Examples," Proc. Advances in Neural Information Processing Systems, pp. 892-900, 2010.

[11] B. Settles, "Active Learning Literature Survey," technical report, 2010.

[12] R. Huang and W. Lam, "Semi-Supervised Document Clustering via Active Learning with Pairwise Constraints," Proc. Int'l Conf. Date Mining, pp. 517-522, 2007. 
[13] P. Mallapragada, R. Jin, and A. Jain, “Active Query Selection for Semi-Supervised Clustering," Proc. Int'l Conf. Pattern Recognition, pp. 1-4, 2008.

[14] Q. Xu, M. Desjardins, and K. Wagstaff, "Active Constrained Clustering by Examining Spectral Eigenvectors," Proc. Eighth Int'1 Conf. Discovery Science, pp. 294-307, 2005.

[15] L. Breiman, "Random Forests," Machine learning, vol. 45, no. 1, pp. 5-32, 2001.

[16] M. Al-Razgan and C. Domeniconi, "Clustering Ensembles with Active Constraints," Applications of Supervised and Unsupervised Ensemble Methods, pp. 175-189, Springer, 2009.

[17] O. Shamir and N. Tishby, "Spectral Clustering on a Budget," J. Machine Learning Research - Proc. Track, vol. 15, pp. 661-669, 2011.

[18] K. Voevodski, M. Balcan, H. Ro“glin, S. Teng, and Y. Xia, "Active Clustering of Biological Sequences,” J. Machine Learning Research, vol. 13, pp. 203-225, 2012.

[19] L. Breiman, "RF/Tools: A Class of Two-Eyed Algorithms," Proc. SIAM Workshop, Statistics Dept., 2003.

[20] T. Shi and S. Horvath, "Unsupervised Learning with Random Forest Predictors," J. Computational and Graphical Statistics, vol. 15, pp. 118-138, 2006.

[21] A. Frank and A. Asuncion, "UCI Machine Learning Repository," http://archive.ics.uci.edu/ml, 2010.

[22] O. Mangasarian, W. Street, and W. Wolberg, "Breast Cancer Diagnosis and Prognosis via Linear Programming," Operations Research, vol. 43, no. 4, pp. 570-577, 1995.

[23] M. Little, P. McSharry, S. Roberts, D. Costello, and I. Moroz, "Exploiting Nonlinear Recurrence and Fractal Scaling Properties for Voice Disorder Detection,” BioMedical Eng. OnLine, vol. 6, no. 1, p. 23, 2007.

\section{AUTHORS}

P. Ganesh Kumar Pursuing my Mtech in JNTUA College in the stream of CSE and done this project under the guidance of Dr.A.P.Siva Kumar. My knowledge and enthusiastic encouragement have impressed me to better involvement into my project thesis and technical design also my guide ethical morals helped me to develop my personal and technical skills to deploy my project in success. Last but far from least, I also thank my family members and my friends for their moral support and constant encouragement, I am very much thankful to one and all who helped me for the successful completion of the project.

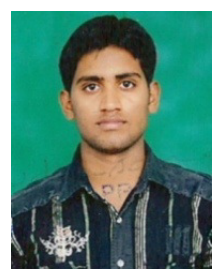

Dr. A. P. Siva Kumar, Assistant Professor of Computer Science and Engineering Department, JNTUA College of Engineering (Autonomous), Ananthapuramu who has extended his support for the success of this project. His wide knowledge and logical way of thinking have made a deep impression on me. His understanding, encouragement and personal guidance have provided the basis for this thesis. His source of inspiration for innovative ideas and his kind support is well to all his students and colleagues. 